\title{
Patient-Controlled Taping for the Treatment of Ingrown Toenails
}

Meiko Tsunoda, $M D^{1}$

Koichi Tsunoda, $M D^{2}$

'Department of Dermatology, Tsunoda Clinic, Tokyo, Japan

${ }^{2}$ Department of Artificial Organs and Medical Device Creation, National Institute of Sensory Organs, National Tokyo Medical Center, National Hospital Organization, Tokyo, Japan

Conflicts of interest: authors report none.

\section{CORRESPONDING AUTHOR}

Koichi Tsunoda

Department of Artificial Organs and Medical Device Creation National Institute of Sensory Organs, National Tokyo Medical Center National Hospital Organization 2-5-1 Higashigaoka, Meguro-ku, Tokyo 159-8902, Japan tsunodakoichi@kankakuki.go.jp

\begin{abstract}
Various methods are used to treat ingrown or pincer-like toenails. We developed a novel taping method to prevent topical interruption of the circulation and resulting skin conditions and evaluated it over 14.5 years. We instructed 541 patients or their guardians in the use of the technique. Ingrown toenail symptoms and abnormal nail growth were resolved and no additional therapy was required in 276 patients. The novel taping method was significantly more effective than treatments our patients had received previously. Patient-controlled taping is the first-line treatment for every ingrown or curved toenail seen in our clinic.
\end{abstract}

Ann Fam Med 2014;12:553-555. doi: 10.1370/afm.1712.

\section{INTRODUCTION}

$\mathrm{V}$ arious methods have been used to treat ingrown or pincer-like (curved) toenails, including taping, ${ }_{1}^{1}$ cotton wool packing, ${ }^{2}$ gutter treatment ${ }^{3}$ with a tube ${ }^{4}$ and with or without formable acrylic, ${ }^{5}$ 3TO (VHO-Osthold brace) treatment, ${ }^{6}$ and surgical procedures. ${ }^{7-10}$ Many patients experience relapse or worsening of ingrown toenails, however, and many visited our clinic after having had primary treatment, especially with excision, elsewhere.

With conventional taping methods, most patients are required to visit a clinic frequently for retaping, and many discontinue taping because of discomfort. In addition, skin irritation from the stretched tape may cause dermatologic problems. Therefore we developed a novel taping method that is easy and comfortable for patients and prevents circulatory problems and dermatologic side effects.

\section{METHODS}

We evaluated the efficacy of the modified taping method we developed in a retrospective observational study, which did not require institutional review board approval. The modifications of the conventional taping procedure are illustrated in Supplemental Figure 1. The elastic adhesive tape used (Elastopore, Nichiban) is wider $(25 \mathrm{~mm})$ and shorter $(3-4 \mathrm{~cm})$ than that used in previous methods. The procedure is explained in the Supplemental Appendix.

While the novel procedure stretches the tape less than $1 \mathrm{~cm}$, in previous methods 1 end of the adhesive tape was fixed to the affected side of the lateral nail fold, and the tape was then stretched, tightened, and pulled over the volar surface of the toe and fixed to the contralateral side. Following the novel procedure correctly results in rapid resolution of pain, usually within 1 week. Patients are advised to change the tape at least once daily and to continue taping until the lateral nail edge is exposed, which usually requires 4 to 8 weeks.

Dampness of the toes due to hyperhidrosis may require additional anchor tapings, as may severe inflammation. Severe cases with infection or granulation may require supplemental procedures with combined oral 
antibiotics or tube (gutter) treatment, and cryosurgery is sometimes necessary when tissue granulation is extensive.

\section{RESULTS}

From December 1996 to July 2011, we instructed 541 patients or their guardians in the use of our novel taping technique. The 541 patients (182 males and 359 females ranging in age from 1 to 93 years, including 28 with diabetes mellitus and some with infection of the ingrown nail) had a total of 750 affected big toenails; 66 had experienced recurrence or worsening of ingrown toenails after surgery or simple excision performed elsewhere. Figure 1 summarizes pertinent information about the 541 patients before and after treatment using our novel method.

We evaluated the results at 2 months after the routine course of taping performed by patients or their guardians. Supplemental Figure 2 shows representative results. The ingrown toenail symptoms and abnormal nail growth were resolved and no additional treatment was required in 276 patients (334 of 750 affected toes, $44.5 \%$ ). The remaining 265 patients (416 of 750 affected toes, $55.5 \%$ ) required additional treatment such as nail bracing or surgery. Still, most of those patients reported relief of pain with taping.

Although some patients discontinued self-care taping treatment because they found it cumbersome or time-consuming, we made an effort to educate them in how and why it was necessary to continue it. Whether their symptoms were resolved, when patients whose toenails were curved requested it, nail brace treatment was applied, although this is not covered by Japanese medical insurance. Those patients tended to discontinue taping, although we recommended that it be continued as supplemental treatment.

As shown in Figure 1, patients whose ingrown toenails had been treated surgically before they visited the author's clinic were more likely to require additional surgery after using the new taping method than those who had not (15/88 affected toes [17.05\%] vs $19 / 662$ $[2.87 \%] ; P<.001$, binominal test $)$.

\section{DISCUSSION}

The results of our retrospective observational study suggest that the novel taping method should be attempted before surgical treatment. Patients who had undergone surgery before using our taping method were more likely to require additional surgery, which may be attributed to the occurrence of postoperative side effects such as nail division, deformity, and/or spicule formation.

Nishioka et al $^{1}$ reported that 11 of 12 patients with ingrown nails responded well to their taping method within 2 to 4 weeks, but recurrence was observed in 2 patients who stopped having their toenails taped after 2 weeks. No recurrence was noted in patients who wore the tape for 2 months. Using another self-care method, an uncontrolled case series found a $79 \%$ (19/25 cases) rate of symptomatic improvement by inserting cotton wool under the ingrowing nail edge over a mean follow-up

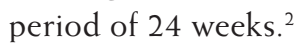

Although 265 of our patients needed additional treatment, the taping relieved pain in the majority. Furthermore, because the length of tape used is approximately 3-4 cm and does not completely encircle the toe, none of 
the 541 patients treated with our novel method experienced topical circulation problems, not even those with diabetes mellitus. Since patients can easily change the tape whenever necessary with this procedure, it is easy to keep the taped toenails clean. We hope that other health care facilities will adopt this novel taping method as a noninvasive, low-cost, first-line treatment for ingrown toenails in the typical primary-care population before cutting or removing the nail.

An important element of our taping treatment is patient education. The taping technique and principle, as well as walking correctly, cutting the toenails square, and wearing appropriate footwear, may prevent the recurrence of ingrown nails. It is necessary to evaluate the additional effects of patient education with the use of this novel taping method, and we plan to investigate this factor in further research.

To read or post commentaries in response to this article, see it online at http://www.annfammed.org/content/12/6/553

Funding support: This research was supported in part by a grant in aid from the National Hospital Organization, Japan.

Acknowledgments: We thank Cynthia Yenches for copyediting multiple versions of this manuscript.

Key words: ingrown nails; self-care; adhesive surgical tape; nail bracing; taping
Submitted February 24, 2014; submitted, revised, August 14, 2014; accepted September 1, 2014.

Supplementary materials: Available at http://www.annfammed. org/content/12/6/553/suppl/DC1/

\section{REFERENCES}

1. Nishioka K, Katayama I, Kobayashi Y, Takijiri C, Nishioka K. Taping for embedded toenails. Br J Dermatol. 1985;113(2):246-247.

2. Senapati A. Conservative outpatient management of ingrowing toenails. J R Soc Med. 1986;79(6):339-340.

3. Wallace WA, Milne DD, Andrew T. Gutter treatment for ingrowing toenails. Br Med J. 1979;2(6183):168-171.

4. Schulte KW, Neumann NJ, Ruzicka T. Surgical pearl: nail splinting by flexible tube-a new noninvasive treatment for ingrown toenails. J Am Acad Dermatol. 1998;39(4 Pt 1):629-630.

5. Arai $\mathrm{H}$, Arai T, Nakajima $\mathrm{H}$, Haneke E. Formable acrylic treatment for ingrowing nail with gutter splint and sculptured nail. Int J Dermatol. 2004;43(10):759-765.

6. Harrer J, Schöffl V, Hohenberger W, Schneider I. Treatment of ingrown toenails using a new conservative method: a prospective study comparing brace treatment with Emmert's procedure. J Am Podiatr Med Assoc. 2005;95(6):542-549.

7. Strube HD, Wasserscheid B. Emmert-plasty for ingrown toenail. Orthoped Traumatol. 1993;2(1):60-65.

8. Zuber TJ. Ingrown toenail removal. Am Fam Physician. 2002;65(12): 2547-2552, 2554.

9. Karaca N, Dereli T. Treatment of ingrown toenail with proximolateral matrix partial excision and matrix phenolization. Ann Fam Med. 2012;10(6):556-559.

10. Heidelbaugh JJ, Lee H. Management of the ingrown toenail. Am Fam Physician. 2009;79(4):303-308.

\section{Get the Annals of Family Medicine by E-mail}

Make sure you see every new issue

while it's fresh; have the table of

contents sent to you by e-mail for

easy access to articles of interest.

Don't miss important research.

Request the e-mail table of contents at

http://lyris.aafp.org/subscribe/annals/.

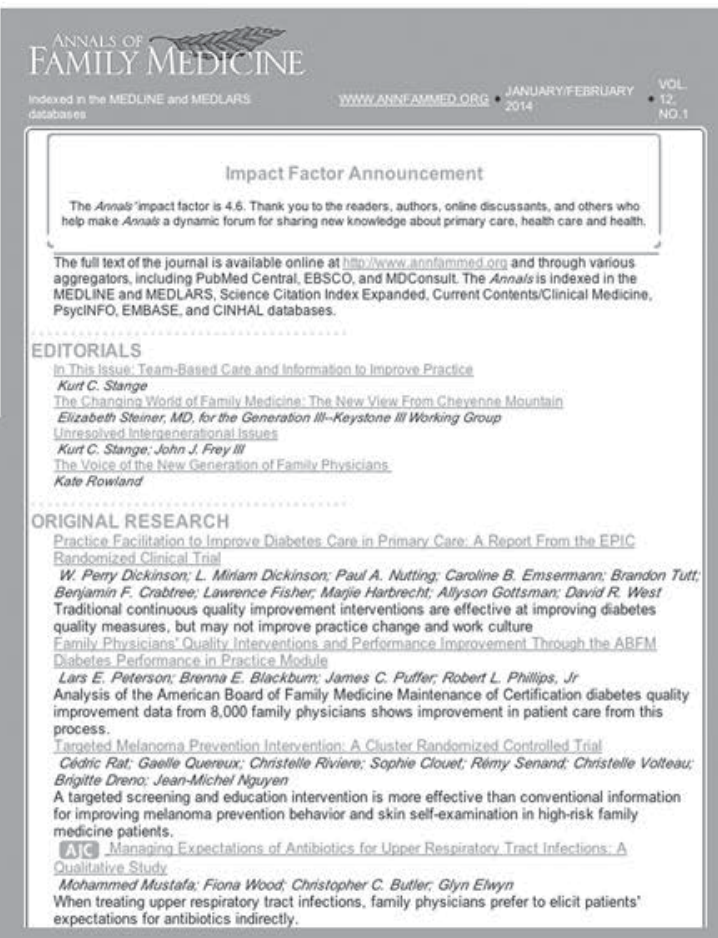

